Jérémie Fischer*

(Ville de) Villefranche-sur-Saône

\title{
Tuberculose, petite vérole et choléra : d'une contamination isolée à la pandémie. Les maladies infectieuses mortelles dans les Mémoires de l'abbé Pochard (1800-1831)**
}

\section{Introduction}

A l'heure où la deuxième vague de la Covid-19 vient de frapper l'Europe, 190 ans ont passé depuis le début de la deuxième pandémie de choléra qui a également ravagé le continent. Ce triste anniversaire, ainsi que la prise de conscience collective de notre vulnérabilité face à ce type d'événement, sont les points de départ de cette brève étude sur les maladies infectieuses mortelles dans les Mémoires de l'abbé Pochard (1766-1833). En plus de ses fonctions de précepteur et gouverneur au sein de la famille du staroste de Gniezno Józef Skórzewski (1757-1809), cet émigré français s'est attelé à rédiger des Mémoires forts intéressants sur la Pologne du premier quart du XIX ${ }^{\mathrm{e}}$ siècle ${ }^{1}$.

* Jérémie Fischer - historien diplômé d'un Master de recherche de l'université de Strasbourg (2008) et d'un Master professionnel en archivistique de l'université de Lyon (2010). Ses travaux historiques portent sur le parcours de l'abbé Pochard (1766-1833). En parallèle il a travaillé comme archiviste municipal. Depuis 2021, il est écrivain-biographe indépendant sous le nom Des racines à la page. Il accompagne des gens désireux de transmettre leur histoire à leurs proches. La sortie de sa biographie de l'abbé Pochard est prévue pour septembre 2022 (www.desracinesalapage.fr); e-mail : jeremie@desracinesalapage.fr. ORCID : 0000-0003-1395-8210.

** Due à la structure de l'article, la rédaction a exceptionnellement décidé de placer les notes de bas de page après le texte principal en tant que notes de fin.

Due to the structure of the article, the editorial staff have exceptionally decided to place footnotes after the main text as endnotes. 
A contre-pied d'une approche chronologique, il s'agit ici d'exhumer le témoignage de ce mémorialiste sur les maladies létales transmissibles de son époque, en élargissant crescendo le spectre de l'étude : partir d'une contamination isolée, poursuivre par une épidémie locale, et finir enfin avec la relation, plus détaillée et plus longue, d'une pandémie ${ }^{2}$ mondiale vue de Pologne. Aussi, l'article s'articule autour des exemples de la tuberculose d'Alexis Lipski en 1822, de l'épidémie de petite-vérole dans le village de Nekla en 1800 et de la pandémie de choléra dans le Royaume du Congrès et le Grand-duché de Posen au cours de l'année 1831. Il est ici question de scruter les réactions et stratégies des individus et des autorités face à un danger invisible, qui n'est pas toujours compris ni pris au sérieux, de la déclaration de la maladie jusqu'au retour à la normale.

\section{Un cas isolé de tuberculose (1822)}

L'un des élèves de l'abbé Pochard est l'orphelin Alexis Lipski, le neveu adolescent de la comtesse Helena Skórzewska (1766-1846) chez qui est employé le prêtre français. De santé fragile, il a possiblement contracté cette maladie chronique plusieurs années auparavant. En effet, dès 1817, il lui était conseillé de prendre les eaux à Bad Reinerz (Duszniki-Zdrój), notamment préconisées pour le traitement des maladies respiratoires ${ }^{3}$. La tuberculose est une maladie infectieuse, contagieuse et mortelle dont l'origine est bactérienne. Connue depuis la Haute Antiquité, elle n'est toujours pas convenablement soignée au début du $\mathrm{XIX}^{\mathrm{e}}$ siècle.

Le 7 mai 1822, le précepteur, la starostine ${ }^{4}$ et sa nièce Maria Lipska se mettent en route pour Częstochowa afin d'y visiter le sanctuaire. Pour la jeune fille, il s'agit d'obtenir la rémission de son frère cadet âgé de seize ans :

Mlle Marie espéroit fort obtenir par l'intercession de la Ste Vierge, renommée avec raison, par les miracles nombreux qu'elle obtient de son fils, en faveur de ceux qui recourent à elle avec confiance et les autres dispositions requises pour cet effet, dans cet endroit appellé en Polonois $\underline{\mathrm{Jasna} \text { Gora }}{ }^{5}$, qui signifie montagne illustre. Melle, espéroit, dis-je, obtenir la guérison parfaite de son cher frère $[\ldots]^{6}$.

Le 9 mai, à peine arrivé à Wrocław, Pochard se rend chez le docteur Remer ${ }^{7}$ à la demande de sa protégée afin d'y prendre des nouvelles du tuberculeux :

Melle Marie m'a prié d'aller m'informer du docteur Remer des nouvelles de son frère, car le médecin Hillbrand avoit promis d'écrire de tems en tems la relation des effets produits par les médecines qu'il avoit donné pour ce malade. Ne l'ayant pas trouvé à la maison, je n'ai pu lui faire aucun rapport ${ }^{8}$. 
Quelques jours plus tard, la compagnie apprend la mort d'Alexis Lipski à Leszno, le 5 mai 1822, à cinq heures du matin. L'adolescent ${ }^{9}$ a succombé à la tuberculose en l'absence de sa sœur, de sa tante et de son précepteur ${ }^{10}$. Deux jours plus tard, il a été enterré dans le caveau familial de Czerniejewo ${ }^{11}$, où il a rejoint ses parents Józefa Lipska née Szołdrska (1780-1811) et Józef Lipski $(1769-1812)^{12}$. Si Pochard ne relate dans ses Mémoires ni la nouvelle du décès ni les funérailles, auxquelles il n'a pas été présent, il raconte néanmoins l'impact de cette mort sur la sœur orpheline d'Alexis. Ainsi, au mois d'août, la starostine décide d'effectuer un nouveau voyage à Dresde ${ }^{13}$, en compagnie de l'abbé et de sa nièce en deuil afin de lui changer les idées :

\begin{abstract}
Mde $^{14}$ la comtesse remarquant que l'espace de deux mois n'avoit pas suffi pour dissiper le chagrin qu'avoit causé à Melle sa nièce, la mort de son frère, dans la crainte qu'elle ne devint tout à fait mélancolique, n'a pas trouvé de meilleur moyen pour dissiper son chagrin que de la mener à Dresde, sous prétexte de la perfectionner surtout dans le chant, s'est déterminée d'y retourner ${ }^{15}$.
\end{abstract}

\title{
II. Une épidémie locale de petite vérole (1800)
}

Deux décennies avant la mort d'Alexis Lipski, et avant même sa naissance, l'abbé Pochard a été confronté à une épidémie de variole. Le 22 août 1800 , le précepteur des Skórzewski et ses hôtes quittent la résidence de Nekla « pour aller voir les jardins sur les frontières de la Silésie ${ }^{16} \|^{17}$. Le lendemain, la compagnie se rend à " Solau $^{18}$ dont le château est grand ${ }^{19}$ " pour y découvrir un jardin qui se trouve être « bien en-dessous de celui de Militsch ${ }^{20}$ quoique dans le même genre ». Après le dîner, les voyageurs prennent le chemin du retour lorsque, en route, ils apprennent que « la petite vérole » s'est déclarée à Nekla. Aussi appelée variole, il s'agit d'une maladie infectieuse, fortement contagieuse et mortelle d'origine virale et épidémique ${ }^{21}$.

La meilleure attitude face à une épidémie et de s'en éloigner et de s'isoler, ce qui n'est pas toujours possible notamment pour des raisons économiques. Heureusement, Pochard est l'employé d'une famille de la noblesse qui possède plusieurs biens tant en Grande-Pologne qu'en Poméranie ainsi qu'une fortune non négligeable. Aussi, il ne leur est pas compliqué de trouver une solution de repli pour rester sains et saufs pendant cette épidémie. L'émigré français raconte : "Après dîner nous sommes revenus sur nos pas jusqu'à Komorze ${ }^{22}$ où la crainte de la petite vérole qui régnoit à Nekla nous a retenu jusqu'au 15 novembre $»^{23}$.

Ainsi, la compagnie a fait demi-tour et s'est temporairement installée dans la résidence secondaire de Komorze, située à une quarantaine de kilomètres au sud de Nekla. Partis pour une excursion de deux jours, les Skórzewski et Pochard ne 
reviennent à la maison que trois mois après, lorsqu'il n'y a plus de danger pour eux $^{24}$. Malheureusement, le mémorialiste ne s'épanche pas sur les conséquences de cette épidémie sur la population du village.

\section{Une pandémie de choléra atteint la Pologne (1831)}

Le XIX ${ }^{\mathrm{e}}$ siècle a été marqué par six pandémies de choléra. Cette maladie épidémique est mortelle à hauteur de 50 à $70 \%$ selon les sources. L'abbé Pochard est le témoin de la deuxième pandémie qui s'est diffusée de 1826 à 1837. Partie du Bengale $^{25}$ en 1826, elle atteint la Perse puis Moscou en 1830. Les soldats russes apportent la maladie dans le Royaume du Congrès ${ }^{26}$ en février 1831 lors de la guerre russo-polonaise débutée par l'insurrection de novembre $1830^{27}$. Après la défaite polonaise d'Ostrołę $\mathrm{ka}^{28}$ au mois de mai, ce sont des insurgés originaires du Grand-duché de Posen ${ }^{29}$ et du Royaume du Congrès fuyant les Russes qui apportent la maladie avec eux en Grande-Pologne.

Habitant du Grand-duché de Posen ${ }^{30}$, l'émigré français passe une dizaine de jours au palais de Czerniejewo à partir de la fin mai 1831. Le 6 juin, il rentre à Komorze où se trouve sa résidence principale depuis une vingtaine d'année ${ }^{31}$. Ce village se trouve à proximité immédiate avec la frontière du Royaume du Congrès. Là, il constate les mesures sanitaires mises en place par les autorités prussiennes pour stopper la pandémie qui vient de pénétrer dans la région:

Depuis plus d'un mois le cordon sur la frontière de Pologne où est Komorze est plus strictement formé à raison dit-on, de la choléra qui s'est déclarée en Pologne ${ }^{32}$, de sorte qu'on ne peut quitter le vilage qu'on habite, sans être muni d'un certificat qui assure qu'on est d'un endroit où cette maladie ne s'est pas déclarée, autrement on est retenu et renvoyé. Si on vient d'un endroit où elle fait des ravages, on est obligé de faire la quarantaine ${ }^{33}$, ce qui interrompt beaucoup les communications. Les soldats montent la garde sous des huttes placées à la distance de 50 pas $^{34}$ les unes des autres, sur toute la frontière prussienne et Pologne ${ }^{35} .{ }^{36}$

A la même période, Pochard relève par écrit le durcissement des mesures de protection et décrit la manière dont les communications ont lieu malgré tout entre les deux provinces :

Au commencement du mois de juin le premier lieutenant des chasseurs Cronstein a quitté Komorze et a été remplacé par un lieutenant de fusiliers, à qui a succédé un autre Zimmermann qui a interrompu entièrement toute communication avec la Pologne, et ce n'est qu'avec peine qu'il a permis au fermier de Komorze ${ }^{37}$ de demander au meunier de Ruda ${ }^{38}$, le cens ${ }^{39}$ qu'il lui doit. Pour cet effet, il s'est rendu avec le 
fermier sur le pont, ayant pris avec lui un pot dans lequel il y avoit du vinaigre ${ }^{40}$, la quittance du fermier a été placée sous le pot et le meunier a mis dans le pot l'argent qu'il devoit et a pris de dessous le pot la quittance et s'est retiré et ce n'est qu'après qu'il a été éloigné que le pot a été pris par le fermier. On parfume avec du vinaigre ou du souffre les gazettes et lettres en les perçant à plusieurs endroits ${ }^{41}$.

C'est vraisemblablement à cause de la pandémie de choléra qui met sa vie en péril et qui l'immobilise à Komorze Przybysłaskie, que Pochard, déjà âgé de 65 ans, rédige son testament le 17 juillet $1831^{42}$. Le lendemain, il revoit la starostine de retour à la maison. Elle lui demande alors de se rendre « à Czerniejewo pour la fête de Mde Raymond ${ }^{43}$ ». Pochard raconte ce déplacement : " Cette fête a été célébrée incognito à raison des circonstances peu favorables où l'on se trouve à raison de la guerre contre les Russes ${ }^{44}$. Il n'y avoit que MM Ignace ${ }^{45}$ et Hilaire ${ }^{46}$ et la nouvelle bonne de Sigismond ${ }^{47}$, Mde Nicolet de Bar-sur-Seine ${ }^{48} \gg{ }^{49}$. On peut se demander si le caractère « incognito » de cette fête, outre la raison politicosociale évoquée, ne se justifie pas avant tout par la pandémie qui a cours ? En effet, organiser des retrouvailles dans ce contexte semble irraisonnable d'autant qu'à 15 kilomètres de là, au nord-est, Gniezno est touchée par le choléra tandis qu'à même distance, au sud-est, c'est Września qui commence également à être infectée ${ }^{50}$.

Le 23 juillet, la compagnie a le plaisir de voir arriver la comtesse Skórzewska. L'abbé, quant à lui, rentre à Komorze le $25^{51}$. En route, il rencontre Ignace qui lui donne notamment des informations sur les règles qui conditionnent la mise en quarantaine :

En passant par Nekla ${ }^{52}$ j'ai vu Mr Ignace qui m'a appris qu'à raison de la choléra, quiconque arrive à Wresnia ${ }^{53}$ de $\operatorname{Posen}^{54}$ ou ses environs de deux milles ${ }^{55}$, doit faire une quarantaine de 10 jours $^{56}$, que Nove Miasto ${ }^{57}$ est cerné comme Posen ${ }^{58}$ et qu'on ne peut passer que difficilement par Milosław ${ }^{59}$ et effectivement la garde est doublée et l'on n'a pas manqué de demander d'où je viens ${ }^{60}$.

Malgré la pandémie, l'émigré français effectue, le 5 août, une excursion qu'il a préméditée depuis quelques temps, non sans arrière-pensées :

Un jour en parlant avec le jardinier des hortensia, il m'a dit que l'officier d'ici l'avoit prié de lui en donner deux pour orner le jardin qu'il a fait sur les frontières, l'idée m'est venue de profiter de cette connoissance pour me procurer le moyen d'apprendre des nouvelles de $\mathrm{Mr}$ Antoine ${ }^{61}$ qui s'est raproché de Komorze en s'établissant à Pyzdry ${ }^{62}$ na Woitostwo ${ }^{63}$, et même la possibilité de le voir, vu qu'il vient assez souvent à Ruda ${ }^{64}$. Pour cet effet j'ai témoigné le désir que j'avois de voir le nouveau jardin, ce qui a fait plaisir à Zimmermann ${ }^{65}$. La pluie abondante qui est tombée et a fait déborder les rivières ${ }^{66}$, ne m'ayant pas permis d'exécuter 
cette promenade aussitôt que je me le proposois, j'ai profité des premiers jours de beau tems pour proposer à l'officier de nous transporter au jardin de sa façon. Il n'a pas hésité d'agréer ma proposition et le 5 août nous nous sommes mis en voiture à 4 heures du soir ${ }^{67}$.

Après la visite du jardin, Pochard cherche à entrer en contact avec son filleul Antoine, sous la supervision stricte de l'officier prussien, qui interdit désormais la transmission de courriers :

[...] le jardinier, qui a apporté deux melons et du jardinage pour Mr Antoine, étant venu auprès de nous et ayant donné son avis ; nous sommes retournés auprès du pont $^{68}$ où il avoit laissé ce qu'il avoit apporté, y étant arrivés, l'officier a fait la visite des melons et du jardinage pour voir s'il n'y avoit point de lettre cachée ; n'y ayant rien trouvé, il a fait porter ces choses sur le pont par ses soldats et a ensuite appellé afin que quelqu'un vint reprendre les melons $\& c^{69}$ et j'ai prié qu'on fit venir le meunier. On m'a appris alors que Mr Antoine est à Ruda et j'ai prié qu'on le fit venir. L'officier qui étoit seul avec moi à la barrière du pont, ayant fait écarter le jardinier, m'a dit de ne parler qu'allemand ou polonois ${ }^{70}$ afin qu'il comprit ce que je dirois et ce qu'on me répondroit. J'ai appris avec plaisir que toute sa famille se porte bien ${ }^{71}$ et un Mr qui étoit venu à la barrière polonoise a annoncé à l'officier qui l'a interrogé sur les nouvelles, lui a répondu qu'il n'y avoit rien de nouveau sinon qu'à Pyzdry la choléra a déjà fait mourir 60 personnes ${ }^{72}$ et ayant témoigné le désir qu'il avoit de nous faire passer le courier (dont nous sommes privés depuis que l'officier est ici) il lui a répondu qu'il ne pouvoit pas le permettre. Après avoir fait faire mes complimens à Mde Antoine ${ }^{73}$, je n'ai pas tardé de me mettre en voiture pour retourner à la maison par un autre chemin $[\ldots]^{74}$.

Au début du mois de septembre, l'ancien précepteur tente de voir son filleul :

Ayant appris que je pourrois facilement voir $\mathrm{Mr}$ Antoine auprès du prame de Komorze, je lui ai fait annoncer que je serois bien aise de lui parler sur les quatre heures le $4.7 \mathrm{bre}^{75}$ je m'y suis rendu quoique le tems fut peu favorable pour un entretien en plein air, vu que toute la matinée il a plu à verse [...]. J'ai profité d'un moment d'interruption de pluie qui n'a recomancé à tomber que lorsque je me rendois dans une barque pour passer la rivière. Heureusement $\mathrm{j}$ 'avois pris la précaution de prendre mon parapluie qui a été cause que le batelier avoit plus de peine et je suis arrivé sur la frontière polonoise où Mr Antoine étoit déjà. Après nous être entretenu pendant quelque tems, malgré la pluie, je l'ai quitté et en passant auprès d'un bâtiment nouveau que les Prussiens ont fait bâtir et qu'on appelle Raste $^{76}$, j'ai été curieux d'y entrer et j'y ai vu une sale au milieu destiné pour les officiers et gardes-frontières prussiens, une petite chambre dont un des côtés est 
garni de fenêtres dans laquelle on reçoit les Polonois qui viennent pour y achetter du sucre, du caffé, de l'arac ${ }^{77}$, du sel \&c on peut se voir et s'entretenir avec eux à travers les vitres et lorsqu'il ont achetté quelque chose, on leur donne en ouvrant une petite fenêtre par laquelle on leur présente un vase de terre dans lequel il y a du vinaigre pour qu'ils y mettent l'argent qu'ils doivent pour la marchandise et un florin de plus pour le vinaigre ${ }^{78}$.

Le 23 septembre, l'abbé est de retour à Komorze après une semaine passée à Czerniejewo au cours de laquelle il a « vu plusieurs officiers prussiens qui ont dîné au château ${ }^{79}$. Pour pouvoir rencontrer l'un de ses anciens élèves qui se trouve de l'autre côté de la frontière, il est à nouveau amené à demander une autorisation spéciale :

A mon arrivée j'ai trouvé un nouvel officier à la maison, à qui j'ai demandé la permission de voir Mr Antoine à Ruda et qu'il m'a accordée très volontiers. Mais je n'ai rien appris de nouveau sinon que son épouse et ses enfants se portent bien ${ }^{80}$.

A partir de la fin du mois de septembre, Pochard accueille successivement Raymond, Ignace et Hilaire, de retour au manoir familial de Komorze :

Le 297 bre Mr Raymond est arrivé à Komorze à son retour des eaux de Karlsbad $^{81}$ qui au lieu de le rétablir lui ont nuit ce qui a été cause du retard de son retour $^{82}$. Il a été obligé de faire la quarantaine de 21 jours $^{83}$ qui lui a coûté assez cher, quoiqu'il n'ait pas eu de commodités étant obligé de rester dans une petite chambre barricadée de manière qu'il ne pouvoit pas sortir. Mr Ignace est arrivé aussi deux jours après lui et enfin Mr Hilaire. Ils ont été ensemble peu de jours car Mr Ignace est reparti le 28 bre jour de l'arrivée de Mr Hilaire. Mr Raymond est parti pour Czerniejewo le 8 8bre $[\ldots]^{84}$.

Le mois suivant, l'épouse de l'un des anciens élèves de l'émigré tombe malade d'une fièvre. Par chance, ce n'est pas un symptôme du choléra :

Le 26 8bre $\mathrm{Mr} \mathrm{Joseph}^{85}$ est venu me voir avec le curé de Kretkowo ${ }^{86}$ et m'a annoncé que Mde Joseph ${ }^{87}$ étoit arrivée à Kretkowo pour être plus à portée du médecin du lazareth ${ }^{88}$ établi dans le château de ce lieu ${ }^{89}$ qui s'est chargé de la guérir entièrement des suites d'une fièvre qui l'avoit mis aux portes du tombeau. M'ayant invité à aller voir son épouse le lendemain, je me suis rendu effectivement à son invitation, mais je n'ai pas trouvé Mr Joseph qui étoit parti pour Broniszewice ${ }^{90}$. [...] De là nous avons rendu visite à l'officier qui préside au lazareth et à sa femme qui a l'air d'une bonne dame et peu de tems après avoir quitté cette famille, composée de 4 personnes savoir deux filles, je suis revenu au logis ${ }^{91}$. 
L'abbé Pochard poursuit ses déplacements malgré la pandémie de choléra dont il ne parle plus dans ses écrits, signe peut-être que les contaminations diminuent. Outre des visites de proches et connaissances, le Français sort aussi de chez lui pour aller célébrer des messes aux côtés de confrères :

[...] [Je] me suis rendu à Komorze, que j'ai quitté de nouveau le $1^{\text {er }}$ nov. pour célébrer la fête, de tous les saints, patronale de Kretkowo ${ }^{92}$, où il n'y a eu que le vicaire de Pogorzelice ${ }^{93}$ qui a eu le sermon, le curé et moi. Nous avons dîné chez Mr Joseph avec les officiers du lazaret. Et après les vêpres des morts j'ai regagné ma retraite d'où je suis parti le 6 pour Pogorzelice pour y célébrer la dédicace de l'église paroissiale, à laquelle ont assisté le curé Pawloski ${ }^{94}$, celui de Kretkowo ${ }^{95}$, celui de Czeszewo ${ }^{96}$ qui a eu le sermon et celui de Dębno ${ }^{97}$ qui est arrivé après l'office. Il y avoit plusieurs laïcs qui sont restés $[\ldots]$ après les vêpres $[\ldots]^{98}$.

La dernière allusion de Pochard concernant le choléra concerne la réouverture de la frontière avec le Royaume du Congrès au début du mois de novembre, indice que la catastrophe sanitaire est sur la fin :

Le 8 9bre Mde la starostine que j'attendois depuis long tems tous les jours, est enfin arrivée à Komorze d'où elle est partie le 10 pour Pyzdry, pour voir la famille de Mr Antoine, vu que la communication entre la Pologne et le duché de Posen est enfin libre $[\ldots]^{99}$.

A la toute fin de novembre, la pandémie de choléra s'est déplacée à l'ouest et l'épidémie cesse dans cette partie du Grand-duché de Posen. Entre le 13 juillet et le 28 novembre 1831, sur les 25211 habitants de la ville de Poznań, 879 sont tombés malades du choléra et 529 en sont décédés (soit $2 \%$ de la population) ${ }^{100}$. Parmi les victimes, on recense le maire de la ville Johann Ludwik Tatzler, ainsi que le feld-maréchal prussien August Neidhardt von Gneisenau. Ce dernier, retraité de l'armée, venait justement de reprendre du service pour organiser la répression contre les patriotes polonais du Grand-duché de Posen ${ }^{101}$. Dans l'ensemble de cette province prussienne qui compte près d'un million d'habitants, 7678 ont succombé à la pandémie soit un peu moins de $1 \%$ de la population. Son voisin, le Royaume du Congrès, aurait subi environ 13000 décès sur 4 millions d'habitants, soit un taux de mortalité de $0,3 \%$.

Le 30 janvier 1832, l'abbé Pochard écrit à son ami, l'abbé Dubulle ${ }^{102}$, qui se trouve en France et lui parle de la pandémie qui vient de sévir dans le Grandduché de Posen ${ }^{103}$. La réponse qui lui parvient le 7 août témoigne de la progression de la maladie jusqu'en France. En effet, son correspondant écrit : « Le choléra nous laisse encore tranquille, on dit qu'il approche Dole ${ }^{104}$, mais il n'y est pas encore et il y a ici bien peu de malade $\gg{ }^{105}$. 
Du Grand-duché de Posen la pandémie s'est propagée en Prusse et a atteint la France en mars 1832. Dans la patrie de l'abbé Pochard 100000 morts environ sont à déplorer, ce qui représente $0,3 \%$ des 32 millions d'habitants du Royaume de France. Le choléra se diffuse aussi au Royaume-Uni, puis en Amérique du Nord et en Amérique centrale. La Chine et le Japon, infectés par d'autres voies, ne sont pas non plus épargnés. La pandémie cholérique suivante se déclarera en 1840 et ne s'éteindra que vingt ans plus tard. Peu de temps avant la pandémie suivante...

\section{Conclusion}

Les trois exemples partagés dans cet article relèvent de maladies différentes, dont les points communs sont leur forte contagiosité et leur taux de mortalité important. A travers des témoignages extraits des Mémoires de l'abbé Pochard, le parti pris était de présenter des niveaux crescendo de contagion : un cas individuel de tuberculose, une épidémie de variole dans un village et une pandémie mondiale de choléra observée depuis la Pologne.

Malgré ces échelles distinctes, un point commun semble avoir émergé. C'est une certaine prise de distance des individus avec les personnes ou les lieux contaminés lorsque cela est possible. Dans le cas lors de la tuberculose d'Alexis Lipski, on constate en effet que ni la starostine, ni Pochard ou encore la sœur du malade ne se trouvent à proximité du malade au moment où il est au plus mal, ce qu'ils auraient pu faire à une distance sécurisée ${ }^{106}$. Pendant l'épidémie de variole à Nekla, l'émigré français et sa compagnie restent à quarante kilomètres de là, le temps nécessaire pour revenir une fois le danger passé. Pour ce qui est de la pandémie de choléra, on a vu que Raymond Skórzewski, qui se trouvait à Varsovie, a fui vers Cracovie puis en Autriche, avant de revenir trop tôt dans le Grandduché de Posen ${ }^{107}$ et d'être contraint à une longue quarantaine. Ces exemples de prise de distance avec les foyers de contagion sont néanmoins à nuancer au regard du statut social et de la richesse de la famille Skórzewski (et par extension de Pochard), qui en font des privilégiés face à ces catastrophes, bien qu'ils puissent eux aussi être victimes de ces maladies comme on l'a vu.

La relation de la pandémie de choléra, quant à elle, a montré un éventail de mesures prises par les autorités qui rappellent celles de nombreux pays touchés récemment par la Covid-19 : fermeture des frontières, mise en place de cordonssanitaires, fermeture des écoles et des théâtres, encadrement des déplacements même à courte distance (avec certificats obligatoires), mobilisation de l'armée, augmentation des contrôles, mise en place de quarantaines et de comportements-barrière (installation de vitres aux guichets, utilisation de vinaigre pour désinfecter), etc.

D'une perspective individuelle et collective, la déferlante de 1831 telle que relatée par l'émigré français recèle de comportements qui peuvent sembler 
contradictoires et qui sont également susceptibles de rappeler certaines réactions dangereuses de 2020/2021. Si la rédaction du testament de l'auteur des Mémoires pendant la pandémie témoigne de sa prise de conscience des risques encourus, d'autres comportements de Pochard et de ses connaissances tendent à montrer une certaine prise de risques. En effet, on observe que le vieil émigré et les membres de sa famille d'accueil (en tout cas ceux présents sur le territoire du Grand-duché de Posen), continuent d'effectuer des petits voyages en passant par des villes infectées, afin de se rendre visite et même de participer à une « fête incognito ». Les risques de contagion liés à ces déplacements en territoires infestés et ces brassages de personnes se produisent aussi pour aller retrouver des connaissance ou célébrer des messes même si la pandémie est alors sur la fin. Là encore, la proximité relationnelle de cette classe supérieure de la société avec les personnes détentrices du pouvoir local leur permet d'obtenir des autorisations de déplacement et de maintenir un semblant de vie normale dans ces circonstances exceptionnelles. Non sans prendre des risques, même si le mémorialiste ne relate aucune contagion due à ces comportements.

La pandémie de choléra de 1831 fait incontestablement écho à celle de la Covid-19 en 2020/2021 : les réactions des contemporains, les stratégies sanitaires des autorités et les comportements individuels et collectifs face à elles se ressemblent sur beaucoup d'aspects. Outre les avancées dans le domaine de la médecine et l'amélioration générale des conditions de vie des populations, la différence majeure entre ces deux crises consiste peut-être, comme l'a noté récemment le médecin Guy Dutau ${ }^{108}$, dans l'aspect multinational de la riposte curative ${ }^{109}$. En effet, comme le dit bien ce dernier : « Désormais, la lutte contre le péril infectieux n'est plus l'affaire d'une ville ou d'une région, comme au temps des épidémies de peste ou des pandémies cholériques. $\gg^{110}$.

\section{Notes de fin}

${ }^{1}$ Trois volumes sur quatre sont consacrés à son exil polonais de 1796 à 1833.

2 Epidémie qui se propage sur plusieurs continents.

${ }^{3}$ Dans ses Mémoires, l'abbé Pochard relate un épisode de 1817 au sujet de la comtesse Skórzewska et de ses neveux Maria et Aleksy Lipski : « [...] son voyage aux eaux de Reinerz, que le médecin Wendt lui avoit conseillé pour elle et pour ses enfants adoptifs [...] », Archiwum Państwowe w Poznaniu (APP), Majątek Czerniejewo-Skórzewscy nº32 (MCS), sygn. 2166, Mémoires polonais, t. 2, p. 387.

${ }^{4}$ Helena Skórzewska était communément appelée starostine car elle était l'épouse de l'ancien staroste de Gniezno.

5 Jasna Góra est le sanctuaire qui accueille des moines paulins.

${ }^{6}$ Biblioteka Uniwersytecka w Poznaniu (BUAMP), rkps sygn. 115 I, Mémoires polonais, t. 3, pp. $17-18$.

7 Wilhelm Hermann Georg Remer (1775-1850), professeur de médecine à Breslau.

${ }^{8}$ BUAMP, rkps sygn. 115 I, Mémoires polonais, t. 3, pp. 20-21. 
${ }^{9}$ Alexis Lipski est né le 14 juillet 1805.

${ }^{10}$ C'est certainement le caractère contagieux et mortel de la tuberculose qui explique que les proches du malade ne l'ont pas veillé jusqu'à la fin.

11 Ville du district de Gniezno en Grande-Pologne, fief à l'époque du général Lipski (17391832), grand-père paternel du défunt. Le caveau familial se trouve dans l'église Saint Jean-Baptiste, où reposent toujours actuellement les Lipski.

12 APP, MCS/2133, copie de l'acte de décès d'Alexis Lipski.

${ }_{13}$ Capitale de la Saxe en Allemagne.

${ }^{14}$ Il s'agit de l'abréviation utilisée par Pochard pour le mot Madame.

15 BUAMP, rkps sygn. 115 I, Mémoires polonais, t. 3, p. 60.

${ }^{16}$ Région historique d'Europe centrale qui se trouve principalement sur le territoire de la Pologne mais également en République tchèque et en Allemagne. En 1831, la majeure partie de la Silésie constitue la province prussienne du même nom, créée en 1815. Sa capitale est Wrocław (Breslau en allemand).

17 BUAMP, rkps sygn. 115 I, Mémoires polonais, t. 1, lettre 8 du 30 décembre 1800, p. 139.

18 Il s'agit de Sułów (Sulau en allemand), village du district de Milicz en Basse-Silésie.

${ }^{19}$ Palais construit en 1680 en style baroque puis remodelé en rococo au XVIII ${ }^{\mathrm{e}}$ siècle.

${ }^{20}$ Milicz, est un chef-lieu de district dans la voïvodie de Basse-Silésie.

${ }^{21}$ La mortalité des malades varie entre 30 et $50 \%$. Cf. B. Różańska-Gambal, Występowanie epidemii ospy prawdziwej na świecie od czasów starożytnych po wspótczesne, « Medycyna Nowożytna »15/1-2, p. 33.

${ }^{22}$ Aujourd'hui désignée sous le nom de Komorze-Przybysławskie. Il s'agit d'un village du district de Jarocin en Grande-Pologne.

${ }^{23}$ BUAMP, rkps sygn. 115 I, Mémoires polonais, t. 1, lettre 8 du 30 décembre 1800, p. 142.

${ }^{24}$ Ibid.

${ }^{25}$ Zone géographique du sous-continent indien partagée entre le Bangladesh et l'Inde.

${ }^{26}$ Etat créé en 1815 lors du traité de Vienne à partir de l'ancien Duché de Varsovie (18071815). Également appelé Royaume de Pologne, son souverain est le tsar de Russie (Nicolas I ${ }^{\text {er }}$ en 1831). Bien qu'autonome et doté d'une constitution libérale c'est un état sous tutelle russe. Sa capitale est Varsovie.

${ }^{27}$ Le soulèvement polonais contre la domination russe dans le Royaume du Congrès a lieu de novembre 1830 à octobre 1831 .

${ }^{28}$ Ville-district dans la voïvodie de Mazovie.

29 Selon Barbara Dettke, ils auraient été 3000 à avoir illégalement passé la frontière du Royaume du Congrès pour prêter main forte au soulèvement patriotique. Cf. B. Dettke, Die Asiatische Hydra : die Cholera von 1830/1831 in Berlin und den preussischen Provinzen Posen, Preussen und Schlesien, Berlin 1995, p. 94.

${ }^{30}$ Province du Royaume de Prusse créée en 1815 lors du traité de Vienne à partir de la zone ouest du Duché de Varsovie.

31 Jusque 1810 environ sa résidence principale était à Nekla.

32 En parlant de « Pologne » l'abbé fait allusion au Royaume du Congrès, aussi appelé Royaume de Pologne, même si dans les fait il s'agit d'une province sous domination russe.

${ }^{33}$ Le principe de précaution qui consiste à placer des personnes en quarantaine remonte à l'antiquité. La durée de quarante jours aurait été définie par Hippocrate au Ve siècle avant notre ère.

${ }^{34}$ Le pas, comme unité de mesure varie selon les lieux et les périodes. Il est généralement compris entre 0,75 et 1,5 mètre, ce qui revient dans le cas présent à voir des gardes tous les 40 à 80 mètres environ.

${ }^{35} \mathrm{La}$ frontière commune entre le Grand-duché de Posen et le Royaume du Congrès s'étend sur 300 kilomètres environ. Mais si l'on ajoute la frontière directe du Royaume de Prusse avec le 
Royaume du Congrès, le total environne les 1000 kilomètres. Considérant un pas à sa valeur minimal et la distance à surveiller, 25000 soldats sont nécessaires, si tant est qu'il n'y en ait qu'un seul par « hutte ». Dans le cas d'un «pas » de 1,5 mètre, la surveillance requiert au minimum 12500 soldats. Selon Jerzy Kozłowski, dès décembre 1830, le nombre de soldats prussiens dans le GrandDuché de Poznań a considérablement augmenté, atteignant environ 30000 . Cf. J. Kozłowski, Wielkopolska pod pruskim zaborem w latach 1815-1918, Poznań 2004, p. 82. Ce cordon militaire a un usage sanitaire mais également politique. En effet, il s'agit aussi d'arrêter, dès leur retour, les résidents du duché partis combattre les Russes dans le Royaume du Congrès.

${ }^{36}$ BUAMP, rkps sygn. 115 I, Mémoires polonais, t. 3, p. 220.

${ }^{37}$ Il pourrait s'agir de Florkowski, exploitant agricole de Komorze dans les années 1820-1830.

${ }^{38}$ Ruda Komorska, village du district de Września en Grande-Pologne. En 1831, il se trouve de l'autre côté de la frontière, sur le territoire du Royaume du Congrès.

${ }^{39}$ Il s'agit d'une redevance.

${ }^{40}$ Le vinaigre était utilisé avec plus ou moins d'efficacité pour désinfecter les mains, les aliments, les lettres ou, comme dans l'exemple présent, les pièces de monnaie.

${ }^{41}$ BUAMP, rkps sygn. 115 I, Mémoires polonais, t. 3, p. 221.

42 APP, MCS/1429.

${ }^{43}$ Maria Skórzewska née Lipska (1804-1888), est la cousine et épouse de Raymond Skórzewski (1791-1859), fils aîné de la starostine. Tous deux ont été élèves de Pochard. Maria a son anniversaire le 16 juillet, d'où l'allusion à sa « fête ».

${ }^{44}$ Depuis le début de la guerre, les forces polonaises ont gagné une dizaine de batailles non décisives contre les Russes. Mais la victoire de ces derniers à la bataille d'Ostrołęka le 16 mai 1831 marque les prémices de l'échec du soulèvement dont elle restera un symbole.

${ }^{45}$ Ignace Skórzewski (1794-1859) est l'un des fils de la comtesse Skórzewska et l'un des anciens élèves de l'émigré français.

${ }^{46}$ Hilaire Skórzewski (1792-1841) est le frère du précédent, ancien élève de l'abbé Pochard.

${ }^{47}$ Zygmunt Skórzewski (1828-1901) est le fils de Raymond et Maria Skórzewski.

${ }^{48}$ Commune française située dans l'Aube.

${ }^{49}$ BUAMP, rkps sygn. 115 I, Mémoires polonais, t. 3, p. 222.

${ }^{50}$ Quelque mois plus tard, au moment de dresser le bilan humain, on comptabilisera 155 morts sur 224 malades à Gniezno et 98 morts sur 190 infectés à Września. Cf. B. Dettke, Die Asiatische Hydra ..., p. 336. Selon elle le choléra arrive à Września en juillet 1831. Ibid., p. 143.

${ }^{51} 30$ kilomètres au sud de Komorze, c'est Pleszew qui est touchée par le choléra à partir de juillet. Sur 461 malades, 252 ne vont pas y survivre. Cf. ibid., pp. 336, 143.

${ }_{52}$ Nekla a beaucoup souffert du choléra en 1831. Pendant la pandémie, les habitants ont prié pour le salut par l'intercession de Notre-Dame de la Consolation de Grodziszczko (village du district de Środa Wielkopolska situé à 8 kilomètres). Les survivants ont fait le vœu de faire un pèlerinage annuel à ce sanctuaire, tradition qui se poursuit de nos jours.

${ }^{53}$ Września est un chef-lieu de district en Grande-Pologne. Elle se trouve à 12 kilomètres de Nekla et 50 de Poznań. La ville est aussi touchée par la pandémie. Voir supra.

${ }^{54}$ Poznań (Posen en allemand) est la capitale historique de la région de Grande-Pologne et la capitale de la voïvodie du même nom. En 1831 c'est la capitale du Grand-duché de Posen. Le choléra s'y est déclaré le 13 juillet 1831. Le premier malade est un soldat de 24 ans du nom de Jablonski, qui décède le lendemain. Cf. B. Dettke, Die Asiatische Hydra ..., p. 141.

${ }^{55}$ Un mille est une unité de mesure qui varie selon les lieux et les époques. Il s'agit vraisemblablement ici du mille prussien qui équivaut à 7,5 kilomètres. La quarantaine s'appliquerait donc à toute personne provenant de Poznań et ses environs dans un rayon de 15 kilomètres.

${ }^{56}$ Le non-respect de cette quarantaine pouvait être puni de la peine de mort. 
${ }^{57}$ Nowe Miasto nad Wartą, village du district de Środa Wielkopolska en Grande-Pologne. Avec Milosław, c'est l'un des lieux de passage, entre Komorze et Poznań.

${ }^{58}$ Dès le 18 juillet 1831 , un cordon sanitaire de 22,5 kilomètres de diamètre est établi autour de la ville de Poznań. Contrairement à ce qu'affirme Pochard, Barbara Dettke prétend que ce cordon sanitaire encercle la ville dans un rayon de 3 milles. Les théâtres et les écoles sont fermées. Les aubergistes ont interdiction de recevoir de nouveaux clients. Cf. B. Dettke, Die Asiatische Hydra ..., p. 142. Selon Olaf Brise, les églises restent néanmoins ouvertes sur demande expresse du roi de Prusse. Cf. O. Brise, Angst in den Zeiten der Cholera : Über Kulturelle Ursprünge des Bakteriums Seuchen-Cordon, Berlin 2003, p. 179.

${ }_{59}$ Ville du district de Września en Grande-Pologne.

${ }^{60}$ BUAMP, rkps sygn. 115 I, Mémoires polonais, t. 3, p. 222.

${ }^{61}$ Antoine Skórzewski (1803-1855) est l'un des fils de la comtesse Skórzewska. Ancien élève de l'abbé Pochard, c'est aussi son filleul.

${ }^{62}$ Pyzdry est une ville du district de Września en Grande-Pologne. En 1831 elle se situe sur le territoire du Royaume du Congrès.

${ }^{63}$ Wójtostwo est un quartier de Pyzdry, situé au sud-ouest du centre-ville.

${ }^{64}$ Ruda Komorska, village du district de Września en Grande-Pologne. Il se situe à 4 kilomètres de Komorze, mais en 1831 il se trouve de l'autre côté de la frontière dans le Royaume du Congrès.

${ }^{65}$ Il s'agit du gradé prussien responsable de cette zone de la frontière. Cf. BUAMP, rkps sygn. 115 I, Mémoires polonais, t. 3, p. 221.

${ }^{66}$ C'est la rivière Prosna qui, à cet emplacement, fait la frontière entre le Grand-duché de Posen et le Royaume du Congrès. A dix kilomètres de là, au nord, elle se jette dans la Warta, à laquelle Pochard fait vraisemblablement aussi allusion.

${ }^{67}$ BUAMP, rkps sygn. 115 I, Mémoires polonais, t. 3, pp. 222-223.

${ }_{68}$ Pont situé à quatre kilomètres au nord de Komorze, au niveau de Ruda Komorska.

${ }^{69}$ Ce signe signifie etc.

${ }^{70}$ Pochard a appris l'allemand pendant son exil suisse (1792-1796) et l'a certainement perfectionné en Grande-Pologne à partir de 1796, tout en apprenant aussi le Polonais.

${ }^{71}$ Antoine est marié à Antonina Hutten-Czapska (1802-1872) depuis 1826. Ils ont deux enfants : Joanna Helena Skórzewska (1827-1898) et Hipolit Skórzewski (1830-1898). Deux autres sont morts en bas âge : Helena Skórzewska (1828-1829) et Mikołaj Prosper Skórzewski (1829-1830).

72 Sur une population d'environ 2000 personnes, ces 60 morts représentent un taux de mortalité à 3\%. Paweł Wietrzykowski, qui a dépouillé les registres paroissiaux de Pyzdry de 1808 à 1837 constate une moyenne de 80 à 90 décès par an sur la période, contre 126, toutes causes confondues, pour la seule année 1831. Cf. Wielkopolskie Towarzystwo Genealogiczne (WTG)/forum/Epidemie $w$ Wielkopolkie, Pawet Wietrzykowski, 20 janvier 2020, (http://wtg-gniazdo.org/forum/viewtopic. php?p=142640).

${ }^{73}$ Antonina est enceinte. Elle accouchera quatre jours plus tard d'un garçon prénommé Władisław (1831-1859).

${ }^{74}$ BUAMP, rkps sygn. 115 I, Mémoires polonais, t. 3, pp. 224-226.

757 bre signifie septembre.

${ }^{76}$ Cette auberge (Rasthaus en allemand) dépend du bureau prussien des douanes. Elle se situe sur le territoire du village de Nowa Wieś Podgórna.

${ }_{77}$ Nom d'un spiritueux obtenu par distillation de canne à sucre ou de riz fermenté.

${ }_{78}^{7}$ BUAMP, rkps sygn. 115 I, Mémoires polonais, t. 3, 227-229.

${ }^{79}$ Ibid., p. 229.

${ }^{80}$ Ibid., p. 230. 
${ }^{81}$ Il s'agit de Karlovy Vary (Karlsbad en allemand), station thermale en République tchèque où Pochard s'est lui-même rendu dix ans auparavant. Cf. APP, MCS/2166, Mémoires polonais, t. 2, Voyages faits en 1821, p. 689.

${ }_{82}$ Avant « Karlsbad», Raymond se trouvait à Varsovie pour une raison inexpliquée. Peut-être a-t-il participé à l'insurrection ? Quoi qu'il en soit, l'aîné des anciens élèves de Pochard a quitté la capitale vers le mois de mai, vraisemblablement pour fuir l'épidémie (et/ou les Russes) pour se rendre à Cracovie avant de rejoindre « Karlsbad». Cf. BUAMP, rkps sygn. 115 I, Mémoires polonais, t. 3, p. 220.

${ }^{83}$ Cette quarantaine, plus longue que celle imposée à Poznań, s'explique peut-être parce qu'il revient de l'étranger.

${ }^{84}$ BUAMP, rkps sygn. 115 I, Mémoires polonais, t. 3, pp. 230-231.

${ }^{85}$ Joseph Skórzewski (1798-1855) est l'un des fils de la comtesse Skórzewska. Il a été l'élève de l'abbé Pochard.

${ }^{86}$ Kretków, village du district de Jarocin en Grande-Pologne. Il appartenait à la famille Skórzewski depuis la fin du XVIII ${ }^{\mathrm{e}}$ siècle. En 1831, le curé de la paroisse est Onufry Ziemkiewicz (né en 1800). Cf. WTG/Wielkopolscy Księża (WK), (http://www.wtg-gniazdo.org/ksieza/main.php).

87 Jozefa Gertruda Katarzyna Niemojowska (1801-1871) est l'épouse de Joseph Skórzewski depuis 1820 .

${ }^{88}$ Un lazaret est un établissement où l'on mettait en quarantaine les malades contagieux. En allemand et en russe ce mot signifie aussi hôpital militaire, mais étant donné le contexte il s'agit vraisemblablement ici du premier sens.

${ }^{89}$ Le palais de Kretków, propriété des Skórzewski aujourd'hui en ruines.

90 Village du district de Pleszew en Grande-Pologne.

${ }^{91}$ BUAMP, rkps sygn. 115 I, Mémoires polonais, t. 3, pp. 231-232.

${ }_{92}$ L'église de Kretków, fondée par Józef Skórzewski en 1802, est dédié à Tous les Saints.

93 Pogorzelica, village du district de Jarocin en Grande-Pologne. Le vicaire de cette paroisse en 1831 est Tomasz Dębiński » (né en 1807). Cf. WTG/WK.

94 Jan Pawłowski (1797-1841). Ordonné en 1821, il est curé de Pogorzelica à partir de 1828. Ibid.

95 Voir note 86.

96 Village du district de Września en Grande-Pologne.

${ }^{97}$ Village du district de Środa Wielkopolska, en Grande-Pologne. Le curé de la paroisse de Dębno en 1831 est Tomasz Cieśliński (1801-1870). Cf. WTG/WK.

${ }^{98}$ BUAMP, rkps sygn. 115 I, Mémoires polonais, t. 3, p. 233.

99 Ibid., p. 234.

${ }^{100}$ Cf. B. Dettke, Die Asiatische Hydra ..., p. 209.

101 August Neidhardt von Gneisenau (1760-1831) est un militaire prussien qui avait combattu les armées napoléoniennes et ses alliés. Retraité, il reprend du service en mars 1831 après le début de l'insurrection à Varsovie. Il meurt du choléra le 23 août 1831, dans son quartier général de Poznań, à l'hôtel de Vienne.

102 Jean-Charles Dubulle (1759-1834) est un prêtre apparenté à l'abbé Pochard par son demi-frère.

${ }^{103}$ Date de réponse marquée sur la lettre de Dubulle du 21 décembre 1831. Cf. APP, $\mathrm{MCS} / 1009$.

${ }^{104}$ Commune du Jura.

105 APP, MCS/1009, lettre du 11 juillet 1832.

${ }^{106}$ Cela étant dit, rien ne montre qu'ils ne se soient pas rendus au chevet du tuberculeux dans les semaines précédentes. Malheureusement, les pages des Mémoires de l'abbé Pochard qui auraient pu relater ces faits sont lacunaires. Cf. BUAMP, rkps sygn. 115 I, Mémoires polonais, t. 3. 107 Vraisemblablement impatient de rejoindre son épouse délaissée. 
${ }^{108}$ Pédiatre-pneumologue, médecin des hôpitaux émérite, professeur associé émérite, ex-chef de service de pédiatrie à Toulouse. Cf. G. Dutau, Quarantaines - De la peste et du choléra au coronavirus Covid-19, « La lettre du Pneumologue » 23 (2020) 1, p. 50.

109 « $[. .$.$] en peu de temps l'identification du virus et le partage des connaissances sur ses$ séquences ont permis de mettre en œuvre des travaux multinationaux pour préparer des vaccins », Ibid., p. 52.

110 Ibid.

\section{Tuberculosis, Smallpox and Cholera: from Isolated Contamination to Pandemic: Deadly Infectious Diseases in the Memoirs of Father Pochard (1800-1831)}

\section{Summary}

The article presents three examples from the writings of the French émigré - Father Pochard. Through individual tuberculosis, the smallpox epidemic on a village scale and then the international cholera pandemic observed in Poland, the article shows the reactions of contemporaries. 190 years after the passage of the second cholera pandemic in Poland, it is interesting to note that the health policy of the authorities, but also the individual and collective reactions, closely looked like those we experience today in Europe affected by the second wave of Covid-19.

\section{Keywords}

history of Poland, Duchy of Poznań, Congress Poland, November Uprising, tuberculosis, smallpox, cholera, epidemic, pandemic, Covid-19, the Skórzewski family, the Lipski family, Father Pochard, diaries

\section{Gruźlica, ospa i cholera: od pojedynczego zakażenia do pandemii. Śmiertelne choroby zakaźne we wspomnieniach Księdza Pocharda (1800-1831)}

\section{Streszczenie}

Artykuł przedstawia trzy przykłady z pism francuskiego emigranta - Księdza Pocharda. Ukazuje reakcje współczesnych na pojedynczy przypadek gruźlicy, epidemię ospy w skali wiejskiej, a następnie obserwowaną w Polsce międzynarodową pandemię cholery. 190 lat po przejściu drugiej pandemii cholery w Polsce warto zauważyć, że polityka zdrowotna władz, a także reakcje indywidualne i zbiorowe bardzo przypominają te, których doświadczamy dzisiaj, kiedy Europa jest dotknięta drugą falą Covid-19. 


\section{Słowa kluczowe}

historia Polski, Wielkie Księstwo Poznańskie, Królestwo Kongresowe, powstanie listopadowe, gruźlica, ospa, cholera, epidemia, pandemia, Covid-19, rodzina Skórzewskich, rodzina Lipskich, ksiądz Pochard, wspomnienia

\section{Mots clés}

histoire de la Pologne, Duché de Posen, Royaume du Congrès, Insurrection de Novembre, tuberculose, petite vérole, variole, choléra, épidémie, pandémie, Covid-19, famille Skórzewski, famille Lipski, abbé Pochard, Mémoires

\section{Sources et bibliographie}

Archiwum Państwowe w Poznaniu, Majątek Czerniejewo-Skórzewscy (n932), sygn. 2133, Paszporty i metryki rodziny Lipskich i Skórzewskich i uwolnienie z wojska hr. Rajmunda Skórzewskiego ; 1824-1833.

Archiwum Państwowe w Poznaniu, Majątek Czerniejewo-Skórzewscy (n932), sygn. 2166, Lettres de Claude Antoine Pochard ou Relation de ce qu'il lui est arrivé et de ce qu'il a vu pendant ses différents voyages en Allemagne, Pologne et Prusse, Tom II. Depuis l'an 1817 jusqu'en 1822. À Komorze 1822.

Archiwum Państwowe w Poznaniu, Majątek Czerniejewo-Skórzewscy (n932), sygn. 1009, Korespondencje l'abbé Pochard 1818-1833.

Biblioteka Uniwersytecka w Poznaniu, rkps sygn. 115 I, t. 1 : Lettres de Claude Antoine Pochard ou Relation de ce qu'il lui est arrivé et de ce qu'il a vu pendant ses différents voyages en Allemagne, Pologne et Prusse. Tom I. Depuis l'an 1796 jusqu'en 1817. À Komorze 1822.

Biblioteka Uniwersytecka w Poznaniu, rkps sygn. 115 I, t. 3 : Sans titre, tome 3 des Mémoires polonais de l'abbé Pochard.

Brise O., Angst in den Zeiten der Cholera : Über Kulturelle Ursprünge des Bakteriums Seuchen-Cordon, Akademie-Verlag, Berlin 2003.

Dettke B., Die Asiatische Hydra : die Cholera von 1830/1831 in Berlin und den preussischen Provinzen Posen, Preussen und Schlesien, Walter de Gruyter, Berlin 1995.

Dutau G., Quarantaines - De la peste et du choléra au coronavirus Covid-19, « La lettre du Pneumologue » 23 (2020) 1, pp. 50-55.

Kozłowski J., Wielkopolska pod pruskim zaborem w latach 1815-1918, Poznań 2004.

Różańska-Gambal B., Występowanie epidemii ospy prawdziwej na świecie od czasów starożytnych po współczesne, « Medycyna Nowożytna » 15 (2008) 1-2, pp. 31-59.

Wielkopolskie Towarzystwo Genealogiczne/forum/Epidemie $w$ Wielkopolsce, Pawet Wietrzykowski, 20 janvier 2020, (http://wtg-gniazdo.org/forum/viewtopic.php? $\mathrm{p}=142640$ ).

Wielkopolskie Towarzystwo Genealogiczne, Wielkopolscy Księża, (http://www.wtg-gniazdo.org/ksieza/main.php). 